\title{
Hydrogenation of the intermediate valence ternary stannides CeRhSn and CeIrSn
}

\author{
B. Chevalier ${ }^{1,}$, C. P. Sebastian ${ }^{2}$, R. Pöttgen ${ }^{2}$ \\ ${ }^{1}$ Institut de Chimie de la Matière Condensée de Bordeaux (ICMCB), CNRS [UPR 9048], \\ Université Bordeaux 1, Avenue du Docteur A. Schweitzer, 33608 Pessac Cedex, France \\ ${ }^{2}$ Institut für Anorganische und Analytische Chemie and NRW Graduate School of Chemistry, \\ Universität Münster, Corrensstrasse 30, D-48149 Münster, Germany \\ * Corresponding author: chevalie@,icmcb.u-bordeaux1.fr
}




\begin{abstract}
CeRhSn and CeIrSn were synthesized from the elements via arc-melting. A single crystal from the CeIrSn sample was investigated on the basis of X-ray diffractometer data: $\mathrm{ZrNiAl}$ type, $P \overline{6} 2 m, a=743.8(2), c=407.58(7) \mathrm{pm}, \mathrm{w} R 2=0.0308,352 \mathrm{~F}^{2}$ values and 14 variable parameters. An important structural feature of CeIrSn are relatively short $\mathrm{Ce}-\mathrm{Ir}$ distances $(4 \times$ 303 and $1 \times 308 \mathrm{pm})$. Hydrogenation of these ternary stannides leads to the formation of the new hydrides $\mathrm{CeRhSnH}_{0.8}$ and $\mathrm{CeIrSnH}_{0.7}$ which adopt the same crystal symmetry as the original compounds but a larger unit cell volume after hydrogen insertion. Magnetization, electrical resistivity and thermoelectric power measurements reveal a change of the ground state of the cerium from intermediate valence to the nearly trivalent state induced by hydrogenation. Moreover, ${ }^{119} \mathrm{Sn}$ Mössbauer spectroscopic data of CeRhSn, CeIrSn and their hydrides at 4.2 and $78 \mathrm{~K}$ indicate only one tin site. The isomer shifts are slightly larger for the hydrogenated samples suggesting a slightly higher $s$ electron density at the tin nuclei of the hydrides.
\end{abstract}

Keywords: Cerium; Hydrogenation; Crystal structure; Magnetism 


\section{Introduction}

Last five years, several investigations were devoted to the hydrogenation of the ternary compounds CeMX ( $\mathrm{M}=$ transition metal and $\mathrm{X}=\mathrm{p}$-element $)$ crystallizing in the hexagonal ZrNiAl-type. The insertion of hydrogen in these structures induces : (i) a structural transition from the hexagonal $\mathrm{ZrNiAl}$-type to the hexagonal $\mathrm{AlB}_{2}$-type during the hydrogenation of $\mathrm{CeNiAl}$ [1] or CeNiGa [2-4] and (ii) a preservation of the crystal symmetry when the hydrides deriving from CeNiIn [5-7], CeRhIn [8, 9] or CePdIn [10] are obtained. Steric consideration can be advanced in order to explain the results concerning the structural properties of these hydrides $\mathrm{CeMXH}_{\mathrm{y}}$; if $\mathrm{M}$ and $\mathrm{X}$ exhibit a small atomic radius (i. e. $\mathrm{Ni}$ and $\mathrm{Al}$ or $\mathrm{Ni}$ and $\mathrm{Ga}$ ), the intermetallic and its hydride show a different crystal structure.

The investigation of the hydrides $\mathrm{CeNiInH}_{\mathrm{y}}[5,6], \mathrm{CeRhInH}_{0.55}[8,9]$ and $\mathrm{CePdInH}_{1.0}[10]$ by X-ray or neutron powder diffraction reveals that $\mathrm{H}$ - or D- atoms are inserted in the $\left[\mathrm{Ce}_{3} \mathrm{M}\right]$ tetrahedral sites. Generally these sites are not fully occupied excepted in the $\mathrm{CeNiInH}_{\mathrm{y}}$ system where the saturated hydride $\mathrm{CeNiInH}_{1.33}$ can be obtained [6]. This last hydrogen occupation induces a pronounced anisotropic expansion of the unit cell; the $a$ parameter decreases (- 3.0 $\%)$ whereas the $c$ parameter increases strongly $(+16.4 \%)$ [5]. These structural changes lead to both the occurrence of very short interatomic H-H distances $(\cong 1.61 \AA)$ violating the "2 $\AA$ rule" for $\mathrm{H}-\mathrm{H}$ separation in metal hydrides and strong bonds between $\mathrm{Ni}$ and $\mathrm{H}$ along the $c$ axis $[7,11]$.

Moreover, the hydrogenation of the CeMX compounds induces interesting physical properties linked to the modification of the valence of cerium : (i) a reduction of the Kondo temperature is observed in the sequence $\mathrm{CeRhIn} \rightarrow \mathrm{CeRhInH}_{0.55}[8,9]$; (ii) a transition from intermediate valence to magnetic behaviour without long-range order is evidenced by $\mathrm{H}$ insertion in $\mathrm{CeNiGa}$ [3]; (iii) a transition from an intermediate valence state to a ferromagnetic behaviour is found in the system $\mathrm{CeNiInH}_{\mathrm{y}}$ [5]; and finally (iv) an increase of the Néel temperature $(1.65 \mathrm{~K} \rightarrow 3.0 \mathrm{~K})$ is detected in the sequence CePdIn $\rightarrow \mathrm{CePdInH}_{1.0}$ [10]. All results can be interpreted considering that the hydrogenation of the compounds based on cerium increases the unit cell volume leading to a reduction in the coupling constant $\mathrm{J}_{\mathrm{cf}}$ between $4 \mathrm{f}(\mathrm{Ce})$ and conduction electrons [3]. This reduction favours the occurrence of a magnetic ordering for cerium in these hydrides.

In this view, it is interesting to study the influence of the hydrogenation on the structural and physical properties of the ternary stannides CeRhSn and CeIrSn, which adopt the hexagonal $\mathrm{ZrNiAl}$-type structure and are classified as the valence-fluctuating systems [12-15]. 
CeRhSn has intensively been investigated ( $a c$ and $d c$ susceptibility, resistivity, and thermopower measurements as well as by ${ }^{119} \mathrm{Sn}$ Mössbauer) in recent years with respect to the outstanding magnetic properties. The structure of CeRhSn was refined from single crystal Xray as well as neutron powder diffraction data, and the electronic structure was elucidated on the basis of TB-LMTO band structure calculations [14, 16-18]. In contrast, only little information is available on CeIrSn. The lattice parameters of this stannide have been reported by Salamakha et al. [19] on the basis of Debye-Scherrer data, revealing isotypism with CeRhSn. CeIrSn has been studied by Bando et al. [12] by electrical resistivity and thermopower measurements. These data indicate an intermediate cerium valence also for CeIrSn. So far, no magnetic data are available for this stannide.

During our systematic studies of equiatomic CeMX intermetallics $(\mathrm{M}=$ late transition metal, $X=$ element of the $3^{\text {rd }}$ or $4^{\text {th }}$ main group) [20-25 and ref. therein], we have reinvestigated CeIrSn in more detail. Herein we report on an X-ray single crystal structure refinement, the first magnetic characterization, and ${ }^{119} \mathrm{Sn}$ Mössbauer spectroscopic data. The main topic of our work was then the hydrogenation of both CeRhSn and CeIrSn in order to study the influence on the cerium valence.

\section{Experimental details}

Starting materials for the preparation of the CeRhSn and CeIrSn samples were cerium ingots (Johnson Matthey), rhodium and iridium powder (Heraeus), and tin granules (Merck), all with stated purities better than $99.9 \%$. In a first step, the moisture sensitive cerium ingots were cut under paraffin oil, washed with $n$-hexane and stored in Schlenk tubes under argon. The paraffin oil and $n$-hexane have been dried over sodium wire and the argon was purified before over titanium sponge $(900 \mathrm{~K})$, silica gel, and molecular sieves.

For both synthesis, the rare earth pieces were first arc-melted [26] to small buttons of about $500 \mathrm{mg}$. The pre-melting procedure strongly reduces shattering during the subsequent reactions with rhodium (iridium) and tin. The cerium buttons were then weighed in the 1:1:1 atomic ratio with cold-pressed pellets $(\varnothing 6 \mathrm{~mm})$ of the rhodium (iridium) powder and pieces of the tin granules. These mixtures were arc-melted under an argon pressure of ca. 600 mbar. The resulting buttons were turned over and remelted three times in order to ensure homogeneity. The total weight losses after the various melting procedures were always smaller than 0.5 weight- $\%$. CeRhSn and CeIrSn were obtained in amounts of ca. $1 \mathrm{~g}$. The powdered samples are dark gray. Single crystals exhibit metallic luster. 
The purity of the samples was checked through Guinier powder patterns in a Guinier

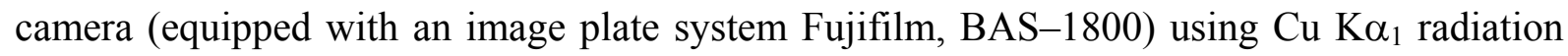
and $\alpha$-quartz $(a=491.30, c=540.46 \mathrm{pm})$ as an internal standard. The hexagonal lattice parameters (Table 1) were obtained by least-squares refinements of the powder data. The correct indexing of the diffraction lines was ensured by intensity calculations [27] using the positional parameters obtained from the structure refinement. The lattice parameters obtained for the powders and the single crystals agreed well. Our data of CeRhSn $(a=744.8(2), c=$ 408.00(9) pm) and CeIrSn (Table 1) are in good agreement with the data published previously: $a=745.8(1), c=408.62(9) \mathrm{pm}$ for CeRhSn [18] and $a=744.9(4), c=408.0(2)$ pm for CeIrSn [19].

Small, irregularly-shaped single crystals of CeIrSn were isolated from the crushed arcmelted sample. The crystals were first examined with white Mo radiation on a Buerger precession camera (equipped with an image plate system, Fujifilm, BAS-1800) in order to establish suitability for intensity data collection. Single crystal intensity data were collected at room temperature by use of a four-circle diffractometer (CAD4) with graphite monochromatized $\mathrm{Mo} \mathrm{K}_{\alpha}(71.073 \mathrm{pm})$ radiation and a scintillation counter with pulse height discrimination. The scans were taken in the $\omega / 2 \theta$ mode and an empirical absorption correction was applied on the basis of psi-scan data, followed by a spherical absorption correction. All relevant crystallographic data and details for the data collection are listed in Table 1.

The CeIrSn single crystal investigated on the four-circle diffractometer and the bulk CeIrSn sample were analysed using a LEICA $420 \mathrm{I}$ scanning electron microscope with $\mathrm{CeO}_{2}$, Ir, and Sn as standards. No impurity elements heavier than sodium were observed. The composition determined by EDX (31(3) at.-\% Ce; 36(3) at.-\% Ir; 33(3) at.-\% Sn) is in good agreement with the ideal 1:1:1 composition. The standard uncertainties arise from the irregular surface of the crystal and the bulk sample.

Hydrogen absorption experiments were performed using the apparatus described previously [28]. An annealed ingot of each ternary stannides was heated under vacuum at 523 $\mathrm{K}$ for $12 \mathrm{~h}$ and then exposed to $4 \mathrm{MPa}$ of hydrogen gas at the same temperature. The hydrogenation induces a decrepitation of the starting ingots. The amount of $\mathrm{H}$ absorbed was determined volumetrically by monitoring pressure changes in a calibrated volume. Under these conditions, the new hydrides $\mathrm{CeRhSnH}_{0.8(1)}$ and $\mathrm{CeIrSnH}_{0.7(1)}$ were obtained. Moreover, these hydrides are stable in ambient conditions. X-ray powder diffraction with the use of a 
Philips 1050-diffractometer $\left(\mathrm{Cu} \mathrm{K} \mathrm{K}_{\alpha}\right.$ radiation) was applied for the characterization of the structural properties of these hydrides.

For transport measurements, the hydrides were compacted at room temperature (compactness $\cong 80 \%$ ) in order to form a polycrystalline pellet (diameter $=6 \mathrm{~mm}$ and thickness $=3 \mathrm{~mm}$ ) and then heated for two days at $523 \mathrm{~K}$ under pressure $(4 \mathrm{MPa})$ of hydrogen. Thermoelectric power measurements were performed on this pellet using a dynamic method. Details of the cell used and measurement methods have been described previously [29]. For electrical resistivity, a bar of $1.5 \times 1.5 \times 5 \mathrm{~mm}^{3}$ was cut from the pellet. The measurement was carried out above $4.2 \mathrm{~K}$ using the standard $d c$ four probe method with silver paint contacts and an intensity current of $10 \mathrm{~mA}$. Finally, magnetization measurements were performed on a part of the pellet using a Superconducting Quantum Interference Device (SQUID) magnetometer in the temperature range 1.8-300 $\mathrm{K}$ and applied fields up to $5 \mathrm{~T}$.

$\mathrm{A} \mathrm{Ca}^{119 \mathrm{~m}} \mathrm{SnO}_{3}$ source was available for the ${ }^{119} \mathrm{Sn}$ Mössbauer spectroscopic investigations. The samples were placed within thin-walled PVC containers at a thickness of about $10 \mathrm{mg}$ $\mathrm{Sn} / \mathrm{cm}^{2}$. A palladium foil of $0.05 \mathrm{~mm}$ thickness was used to reduce the tin $\mathrm{K} \mathrm{X}$-rays concurrently emitted by this source. The measurements were conducted in the usual transmission geometry at 78 and $4.2 \mathrm{~K}$.

\section{Results and discussion}

\subsection{Structure Refinement of CeIrSn}

Isotypy of the crystal with CeRhSn [18] was already evident from the Guinier powder pattern. The atomic positions of CeRhSn were then taken as starting values and the structure was refined using SHELXL-97 (full-matrix least-squares on $F^{2}$ ) [30] with anisotropic atomic displacement parameters for all atoms. The occupancy parameters were refined in a separate series of least-squares cycles. All sites were fully occupied within one standard uncertainty. Refinement of the correct absolute structure was ensured through refinement of the Flack parameter [31,32]. A final difference electron-density synthesis was flat and did not reveal any significant residual peaks. The results of the structure refinement are summarized in Table 1. The atomic coordinates and the interatomic distances are listed in Tables 2 and 3. Further information on the structure refinement is available.*

* Details may be obtained from: Fachinformationszentrum Karlsruhe, D-76344 EggensteinLeopoldshafen (Germany), by quoting the Registry No. CSD-\#\#\#\#\# (CeIrSn). 
Our single crystal X-ray data of CeIrSn clearly confirm the X-ray powder investigation by Salamakha et al. [19]. CeIrSn crystallizes with the ZrNiAl structure, space group $P \overline{6} 2 m$, a ternary ordered version of the well known $\mathrm{Fe}_{2} \mathrm{P}$ type [33-35]. Since the crystal chemistry of isotypic CeRhSn [18] and other ZrNiAl type intermetallics [36] has been discussed in detail, here we only focus on some peculiarities of CeIrSn. Also for drawings of the structure we refer to [18].

The course of the lattice parameters of the series REIrSn $(\mathrm{RE}=\mathrm{La}-\mathrm{Nd}, \mathrm{Sm}, \mathrm{Gd}, \mathrm{Tb}, \mathrm{Ho}-$ $\mathrm{Lu})[19,37]$ already reveals an anomaly for CeIrSn, similar to the series of RERhSn stannides [38]. The $c$ lattice parameter of CeIrSn is even smaller than the $c$ parameter of SmIrSn. This behavior is a direct consequence of the intermediate valence character of the cerium atoms. Since the $c$ lattice parameter of these $\mathrm{ZrNiAl}$ related intermetallics is strongly dominated by the size of the rare earth atoms, the intermediate cerium valence leads to the shrinking of the $c$ parameter.

Accompanied with the shorter $c$ axis are five short Ce-Ir contacts $(4 \times 303$ and $1 \times 308$ pm), only slightly larger than the sum of the covalent radii [39] of $291 \mathrm{pm}$. These Ce-Ir contacts can be considered as significantly bonding. Recent electronic structure calculations of CeRhSn [18] revealed significant $\mathrm{Ce}-\mathrm{Rh}$ bonding interactions. We can safely assume a rigid band model and adopt these bonding characteristics also for CeIrSn.

The iridium and tin atoms in CeIrSn build up a three-dimensional [Ir-Sn] network in which the cerium atoms are located in distorted hexagonal channels. They are connected with the network via the short Ce-Ir contacts. The Ir-Sn distances range from 277 to $283 \mathrm{pm}$, close to the sum of the covalent radii of $266 \mathrm{pm}$ [39]. Within the [Ir-Sn] network we observe Sn-Sn distances of $324 \mathrm{pm}$, slightly longer than in the $\beta$-Sn structure $(4 \times 302$ and $2 \times 318 \mathrm{pm})$ [40]. Finally we need to discuss the slightly enhanced $U_{33}$ parameter of the Ir1 position (Table 2). Also this is a consequence of the intermediate valence character of cerium. Due to the shrinking of the $c$ parameter, the trigonal cerium prism around Ir1 significantly contracts. This behavior is similar to CeRhSn [18]. It is noteworthy that LaRhSn [18] did not show this anomaly.

\subsection{Hydrogenation of CeRhSn and CeIrSn}

3.2.1. Structural properties 
Using X-ray powder diffraction, it was established that the hydrides $\mathrm{CeRhSnH}_{0.8}$ and $\mathrm{CeIrSnH}_{0.7}$ crystallize in the same structure as CeRhSn and CeIrSn (ZrNiAl-type, S.G. P $\overline{6}$ $2 \mathrm{~m}$ ). The hydrogenation of these ternary stannides leads to a rather isotropic expansion of the unit cell (Table 4); the $a$ and $c$ parameters increase in the same manner. In both cases, the unit cell volume V increases. Similar structural changes were reported previously concerning the modest hydrogenation or deuteration of ternary indides CeNiIn $(y(D)=0.48)[6]$, CeRhIn $(y(H)=0.55[9]$ and CePdIn $(y(H)=1.0)[10]$.

It is interesting to compare the unit cell volume of the hydride $\mathrm{CeMXH}_{\mathrm{y}}$ versus the volume of the initial intermetallic CeMX. In figure 1, the dashed line presents the linear fit of the curve $\mathrm{V}\left(\mathrm{CeMXH}_{\mathrm{y}}\right)=\mathrm{f}(\mathrm{V}(\mathrm{CeMX}))$. Three comments can be made from this figure : (i) the data relative to $\mathrm{CeNiInH}_{0.48}$ and $\mathrm{CeRhInH}_{0.55}$ are below the dashed line; it was reported that these hydrides exhibit an intermediate valence behaviour $[9,41]$; (ii) for $\mathrm{CePdInH}_{1.0}$, the data is nearly located on the line; this agrees with the trivalent state of cerium found in CePdIn and its hydride [10]; (iii) finally, the data of the two hydrides $\mathrm{CeRhSnH}_{0.8}$ and $\mathrm{CeIrSnH}_{0.7}$ are well above of the dashed line, suggesting that cerium could be trivalent.

\subsubsection{Magnetic, electrical and thermoelectric properties}

Figure 2 shows the temperature dependence of the reciprocal magnetic susceptibility $\chi_{\mathrm{m}}{ }^{-1}$ of CeRhSn, CeIrSn and their hydrides. The susceptibility of the hydrides is higher than that of the initial ternary stannides.

No Curie-Weiss behaviour is observed for CeRhSn and CeIrSn. Above $100 \mathrm{~K}$, these two curves $\chi_{\mathrm{m}}{ }^{-1}=\mathrm{f}(\mathrm{T})$ have been analysed using the modified Curie-Weiss expression $\chi_{\mathrm{m}}{ }^{-1}=$ $1 /\left(\chi_{0}+\mathrm{C} /\left(\mathrm{T}-\theta_{\mathrm{p}}\right)\right)$ where $\theta_{\mathrm{p}}$ is the asymptotic Curie temperature, $\mathrm{C}$ the Curie constant and $\chi_{0}$ includes the temperature independent Van Vleck correction, the diamagnetic core correction and the paramagnetic contribution of the conduction electrons. The values of $\theta_{\mathrm{p}}, \chi_{0}$ and $\mu_{\mathrm{eff}}=$ $(8 \mathrm{C})^{1 / 2}$ have been refined by a least squares method giving $\theta_{\mathrm{p}}=-61 \mathrm{~K}, \chi_{0}=0.7610^{-3} \mathrm{emu} / \mathrm{mol}$ and $\mu_{\text {eff }}=1.25 \mu_{\mathrm{B}} / \mathrm{Ce}$ for CeRhSn and $\theta_{\mathrm{p}}=-16 \mathrm{~K}, \chi_{0}=0.6110^{-3} \mathrm{emu} / \mathrm{mol}$ and $\mu_{\mathrm{eff}}=1.32$ $\mu_{\mathrm{B}} / \mathrm{Ce}$ for CeIrSn. The effective paramagnetic moment $\mu_{\mathrm{eff}}$ obtained for CeRhSn and CeIrSn is smaller than that calculated for a free $\mathrm{Ce}^{3+}$ ion $\left(2.54 \mu_{\mathrm{B}}\right)$ suggesting an intermediate valence behaviour of cerium in these ternary stannides. Our data concerning CeRhSn are in good agreement with the data reported previously using powder or single-crystal of this ternary stannide [13, 14]. Finally, no distinct anomaly ascribed to the occurrence of magnetic 
ordering was observed near $6.2 \mathrm{~K}$, in contrast to the previous report on a polycrystalline sample [17].

Above $200 \mathrm{~K}$, the curves $\chi_{\mathrm{m}}{ }^{-1}=\mathrm{f}(\mathrm{T})$ relative to $\mathrm{CeRhSnH}_{0.8}$ and $\mathrm{CeIrSnH}_{0.7}$ can be fitted with a Curie-Weiss law $\chi_{\mathrm{m}}{ }^{-1}=\left(\mathrm{T}-\theta_{\mathrm{p}}\right) / \mathrm{C}$ giving respectively $\theta_{\mathrm{p}}=-174 \mathrm{~K}$ and $\mu_{\text {eff }}=2.66 \mu_{\mathrm{B}} / \mathrm{Ce}$ for $\mathrm{CeRhSnH}_{0.8}$ and $\theta_{\mathrm{p}}=-182 \mathrm{~K}$ and $\mu_{\text {eff }}=2.55 \mu_{\mathrm{B}} / \mathrm{Ce}$ for $\mathrm{CeIrSnH}_{0.7}$. The effective moment values are very close to that calculated for a free $\mathrm{Ce}^{3+}$ ion $\left(2.54 \mu_{\mathrm{B}}\right)$. The negative curvature observed at low temperatures in the $\chi_{\mathrm{m}}{ }^{-1}=\mathrm{f}(\mathrm{T})$ curves, indicates the presence of a crystalfield effect. In other words, hydrogenation of the ternary stannides CeRhSn and CeIrSn induces a change of the valence state of cerium from intermediate to purely trivalent. It is interesting to compare these results to those obtained previously on the similar hydrides $\mathrm{CeNiInH}_{0.5}$ and $\mathrm{CeRhInH}_{0.55}[9,41]$. Magnetization measurements performed on these two last hydrides reveal that cerium continues to be in an intermediate valence state. This comparison agrees with the expansion of the unit cell (Fig. 1) reported during the hydrogenation of these ternary indides and stannides.

At low temperatures, an interesting result is derived from comparing the temperature dependence of the magnetic susceptibility of $\mathrm{CeRhSnH}_{0.8}$ measured in zero-field-cooled (ZFC) and field-cooled (FC) modes (Fig. 3). A small irreversibility effect appears below $\mathrm{T}_{\mathrm{IR}}=$ $6 \mathrm{~K}$. This behaviour is a typical feature expected for spin glasses [42]. Similar result was observed recently by us on the hydride $\mathrm{CeNiGaH}_{1.1}$ and was interpreted in terms of shortrange magnetic interactions dictated by crystallographic disorder (the partial occupancy of the crystallographic site devoted to the $H$ atoms, induces structural disorder around the Ce atoms) [3]. More improvements on the magnetic transition of $\mathrm{CeRhSnH}_{0.8}$ will be performed in a near future.

Figure 4 shows the thermal dependence of the reduced electrical resistivity of the two hydrides $\mathrm{CeRhSnH}_{0.8}$ and $\mathrm{CeIrSnH}_{0.7}$. (Due to the presence of many porosities and microcracks resulting from the low temperature $(523 \mathrm{~K})$ used during the annealed treatment performed on these samples, absolute value of $\rho(T)$ could not be determined accurately; for this reason, reduced resistivity is reported). Before explaining these behaviours and in order to understand it's, we should make reference to the electrical resistivity measurements of CeRhSn and CeIrSn. For CeRhSn, the $\rho$ versus $\mathrm{T}$ plot was reported for a polycrystalline sample and single crystals: (i) in the first case, $\rho(\mathrm{T})$ increases with decreasing temperature from $300 \mathrm{~K}$ to $90-100 \mathrm{~K}$ then decreases [17]; (ii) two remarkable features were reported by the measurements performed on single crystals; the occurrence of a large maximum at $70 \mathrm{~K}$ 
when $\rho(\mathrm{T})$ is measured along the $a$-axis and a high anisotropy [13]. These behaviours are characteristic of cerium intermetallics with exhibit valence instabilities. On the contrary, only one reference exists concerning the $\rho$ versus $\mathrm{T}$ plot of CeIrSn [12]; $\rho(\mathrm{T})$ shows a large maximum around 100-120 K.

Electrical resistivity of $\mathrm{CeRhSnH}_{0.8}$ and $\mathrm{CeIrSnH}_{0.7}$ exhibits a different behaviour (Fig. 4). It is interesting to note that the curves $\rho=f(T)$ relative to these two hydrides are practically identical between $290 \mathrm{~K}$ and $35 \mathrm{~K}$. Two features can be observed on the curve relative to $\mathrm{CeRhSnH}_{0.8}$ : (i) below $290 \mathrm{~K}, \rho(\mathrm{T})$ increases with decreasing temperature up to a broad maximum appearing around $17 \mathrm{~K}$; (ii) below this maximum, $\rho(\mathrm{T})$ decreases slowly and more rapidly below $6 \mathrm{~K}$ (inset of Fig. 4) in agreement with the occurrence of short-range magnetic interactions (Fig. 3). Above 50-60 K, the curve $\rho=\mathrm{f}(\mathrm{T})$ of this hydride is characterized by incoherent Kondo scattering with a $\rho=-\mathrm{A} \log \mathrm{T}(\mathrm{A}=$ constant $)$ dependence [43]. We note that the temperature $(17 \mathrm{~K})$ of the maximum of the curve $\rho=\mathrm{f}(\mathrm{T})$ for $\mathrm{CeRhSnH}_{0.8}$ is smaller than that observed $(70 \mathrm{~K})$ for CeRhSn, suggesting a decrease of the influence of the Kondo effect during the hydrogenation of this ternary stannide. Finally, $\rho(\mathrm{T})$ for $\mathrm{CeIrSnH}_{0.7}$ exhibits a negative temperature coefficient in the entire range 290-4.2 K.

The thermal dependence of the thermoelectric power $\mathrm{S}$ of CeRhSn and CeIrSn [12] resembles that observed for $\mathrm{CePd}_{3}$ which is well known as a canonical intermediate valence compound $[44,45]$. In the temperature range $4.2-500 \mathrm{~K}, \mathrm{~S}$ is always positive but presents a shoulder at low temperature $(20-40 \mathrm{~K})$ and a large peak at high temperature; $S=f(T)$ for CeRhSn and CeIrSn has a broad maximum of $60 \mu \mathrm{V} / \mathrm{K}$ at $150 \mathrm{~K}$ and $40 \mu \mathrm{V} / \mathrm{K}$ at $300 \mathrm{~K}$, respectively. These behaviours can be qualitatively understood in terms of the CoqblinSchrieffer model (CSM) which describes the dynamics of conduction electrons due to the exchange and potential scattering on incoherent $4 \mathrm{f}(\mathrm{Ce})$ states [45]. This model uses the crystalline electric field (CF) ( $\Delta$ as energy separation between ground and excited levels) and the number of $4 \mathrm{f}(\mathrm{Ce})$ electrons $\left(n_{f} \leq 1\right.$ for cerium). The curves $\mathrm{S}=\mathrm{f}(\mathrm{T})$ for CeRhSn and CeIrSn are obtained for the (CSM) model with large (CF) spitting and $\mathrm{n}_{\mathrm{f}}<1$ (strong Kondo temperature $T_{K}$ as in intermediate valence compounds).

The curves $\mathrm{S}=\mathrm{f}(\mathrm{T})$ for the hydrides $\mathrm{CeRhSnH}_{0.8}$ and $\mathrm{CeIrSnH}_{0.7}$ show a clear different behavior (Fig. 5). It is mainly characterized for $\mathrm{CeRhSnH}_{0.8}$ by the existence of three extremes: a positive broad maximum of about $19 \mu \mathrm{V} / \mathrm{K}$ near $250 \mathrm{~K}$, a small negative $-1 \mu \mathrm{V} / \mathrm{K}$ around $37 \mathrm{~K}$ and finally a second positive maximum $2 \mu \mathrm{V} / \mathrm{K}$ at $8 \mathrm{~K}$. There is also two changes in sign at 54 and $24 \mathrm{~K}$. Similar change of sign has been observed for nearly trivalent 
cerium-based compounds as $\mathrm{CeCu}_{2} \mathrm{Si}_{2}, \mathrm{CeCu}_{2} \mathrm{Ge}_{2}, \ldots$ [45]. Most of these compounds order magnetically or become superconducting at lowest temperatures. The characteristics of the curves $\mathrm{S}=\mathrm{f}(\mathrm{T})$ for $\mathrm{CeRhSnH}_{0.8}$ can be explained using the (CSM) model $[45,46]$ : (i) $\mathrm{S}$ reaches a zero value at a temperature typically of the order of $3 \mathrm{~T}_{\mathrm{K}}$ (we estimated $T_{K}=54 \mathrm{~K} / 3$ $=18 \mathrm{~K}$ for this hydride); (ii) $\mathrm{S}$ goes through a positive maximum at a temperature depending on (CF) (an increase of $\Delta$ shifts the temperature of this maximum to higher values); (iii) finally at low temperatures, $\mathrm{S}$ changes of sign between $\mathrm{T}_{\mathrm{K}}$ and $2 \mathrm{~T}_{\mathrm{K}}$ (between 18 and $36 \mathrm{~K}$ in agreement with the experimental data of $24 \mathrm{~K}$ ) and shows a maximum around $\mathrm{T}_{\mathrm{K}} / 2=9 \mathrm{~K}$ close to the experimental value of $8 \mathrm{~K}$. The comparison of the curves $\mathrm{S}=\mathrm{f}(\mathrm{T})$ for $\mathrm{CeRhSn}$ and $\mathrm{CeRhSnH}_{0.8}$ evidences that the hydrogenation of the ternary stannide changes the valence state of the cerium from intermediate to purely trivalent.

The curve $\mathrm{S}=\mathrm{f}(\mathrm{T})$ for $\mathrm{CeRhSnH}_{0.8}$ presents many similarities to that reported for the isomorphous ternary stannide CePdSn [47]. In this case also, the strong ( $\cong 100 \mathrm{~K})$ and small ( $2 \mathrm{~K}$ ) positive peaks are separated by the negative peak around $20 \mathrm{~K}$. It is well known that CePdSn can be considered as an antiferromagnetic Kondo system with a Néel temperature $\mathrm{T}_{\mathrm{N}}$ of $7 \mathrm{~K}$. Moreover, the evolution of the curve $\mathrm{S}=\mathrm{f}(\mathrm{T})$ from CeRhSn to $\mathrm{CeRhSnH}_{0.8}$ can be compared to that reported recently by Link et al [48] which have applied isostatic pressure on the ternary silicide $\mathrm{CePd}_{2} \mathrm{Si}_{2}$. Without pressure, $\mathrm{S}$ of this last compound exhibits at low temperature a negative peak followed by a broad positive contribution with increasing temperature. On the contrary, under a pressure of $0.9 \mathrm{GPa}$ two positive contributions located

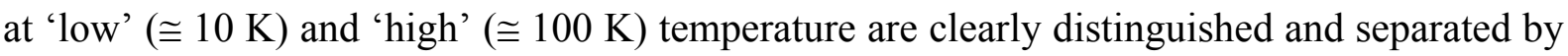
a shallow negative peak ( $\cong 35 \mathrm{~K}$ ); this behavior resembles to that observed here for the hydride $\mathrm{CeRhSnH}_{0.8}$. Finally, at $5.6 \mathrm{GPa} \mathrm{S}$ is always positive showing a shoulder near $20 \mathrm{~K}$ and a strong positive peak around $80 \mathrm{~K}$ with increasing temperature; this behavior is comparable to that reported for the stannide CeRhSn [12]. The transition between these three behaviors is explained by the sequence nearly trivalent cerium $\rightarrow$ intermediate valence cerium induced by the pressure. An opposite sequence is detected here by the insertion of $\mathrm{H}$ atom into CeRhSn. This result confirms that is this case, the hydrogenation can be considered as an application of 'negative' pressure on compounds based on cerium.

No clear positive peak is observed below $300 \mathrm{~K}$ from the curve $\mathrm{S}=\mathrm{f}(\mathrm{T})$ for the hydride $\mathrm{CeIrSnH}_{0.7}$ (Fig. 5). On the contrary, $\mathrm{S}$ changes in sign at $153 \mathrm{~K}$ suggesting that the Kondo temperature of $\mathrm{CeIrSnH}_{0.7}$ is higher than that estimated for $\mathrm{CeRhSnH}_{0.8}$. 


\section{3. ${ }^{119} \mathrm{Sn}$ Mössbauer Spectroscopy}

${ }^{119} \mathrm{Sn}$ Mössbauer spectra of CeRhSn, CeRhSn $0.8, \mathrm{CeIrSn}$, and $\mathrm{CeIrSn}_{0.7}$ taken at $78 \mathrm{~K}$ are presented in Figures 6 and 7 together with transmission integral fits. The fitting parameters of the 78 and $4.2 \mathrm{~K}$ data (not shown in the figures) are presented in Table 5. The low temperature spectra are almost identical with those taken at $78 \mathrm{~K}$ indicating that there are no transferred hyperfine fields at the tin nuclei.

CeRhSn and $\mathrm{CeRhSn}_{0.8}$ (Fig. 6) show single signals subjected to quadrupole splitting $\Delta \mathrm{E}_{\mathrm{Q}}$ due to the non-cubic site symmetry $(\mathrm{m} 2 \mathrm{~m})$ of the tin site. At 78 and $4.2 \mathrm{~K}, \Delta \mathrm{E}_{\mathrm{Q}}$ increases in the sequence $\mathrm{CeRhSn} \rightarrow \mathrm{CeRhSnH}_{0.8}$ in agreement with the presence of $\mathrm{H}$-atoms surrounding the Sn nuclei. In contrast, $\Delta \mathrm{E}_{\mathrm{Q}}$ for CeIrSn is less pronounced (Fig. 7). A refinement with variable line width and a variable quadrupole splitting parameter was not stable. The best refinement was obtained without quadrupole splitting and a variable line width. The small quadrupole splitting of CeIrSn then manifests itself through the slightly enhanced line width when compared with the rhodium containing samples. The hydrogenated sample CeIrSnH $\mathrm{H}_{0.7}$ then reveals larger quadrupole splitting, indicating a different electronic situation of the tin site in $\mathrm{CeIrSnH}_{0.7}$.

The isomer shifts of the samples range from 1.75 to $1.87 \mathrm{~mm} / \mathrm{s}$. This is the usual range for tin in intermetallic compounds [49-51]. Both hydrogenated samples show a slightly higher isomer shift at 4.2 and $78 \mathrm{~K}$, respectively, indicating a slightly higher $s$ electron density at the tin nuclei. Similar behaviour has recently also been observed for the stannides $\operatorname{CaMSn}_{2}(\mathrm{M}=$ $\mathrm{Rh}, \mathrm{Pd}, \mathrm{Ir})$ [52]. In view of the standard uncertainties, however, the increase in line width should not be overinterpreted.

\section{Conclusion}

H-insertion into the ternary stannides CeRhSn and CeIrSn : (i) allows to obtain the new hydrides $\mathrm{CeRhSnH}_{0.8}$ and $\mathrm{CeIrSnH}_{0.7}$ which crystallize with the same crystal symmetry as the parent compounds: (ii) induces an increase of the unit cell volume, 4.4 and $4.7 \%$ in the case of CeRhSn and CeIrSn respectively; (iii) a change of the ground state of the cerium from intermediate valence to the nearly trivalent state. This last modification is correlated to an increase of the unit cell volume induced by hydrogen insertion which leads to a decrease of the hybridisation between $4 \mathrm{f}(\mathrm{Ce})$ electrons and conduction electrons. In other words, the hydrogenation can be compared to the application of a 'negative' pressure inducing a 
decrease of the influence of the Kondo effect on the physical properties of these ternary stannides.

\section{Acknowledgments}

Stimulating discussions concerning the transport properties of the hydrides with Dr. B. Coqblin (Orsay, France) are gratefully acknowledged. We thank U. Ch. Rodewald and B. Heying for the single crystal data collection and H. J. Göcke for the work at the scanning electron microscope. This work was financially supported by the Deutsche Forschungsgemeinschaft. C.P.S. is indebted to the NRW Graduate School of Chemistry for a $\mathrm{PhD}$ stipend. Finally, one of us (B. C.) wish to thank European Science Fundation (ECOMCOST action P16) for financial support. 


\section{References}

[1] J.-L. Bobet, B. Chevalier, B. Darriet, M. Nakhl, F. Weill, J. Etourneau, J. Alloys Comp., 317-318 (2001) 67.

[2] B. Chevalier, J.-L. Bobet, E. Gaudin, M. Pasturel, J. Etourneau, J. Solid State Chem., 168 (2002) 28.

[3] B. Chevalier, J. Sanchez Marcos, J. Rodriguez Fernandez, M. Pasturel, F. Weill, Phys. Rev. B, 71 (2005) 214437.

[4] M. Stange, V. Paul-Boncour, M. Latroche, A. Percheron-Guégan, O. Isnard, V. A. Yartys, J. Alloys Comp., 404-406 (2005) 144.

[5] B. Chevalier, M. L. Kahn, J.-L. Bobet, M. Pasturel, J. Etourneau, J. Phys. : Condens. Matter, 14 (2002) L365.

[6] V. A. Yartys, R. V. Denys, B. C. Hauback, H. Fjellvag, I. I. Bulyk, A. B. Riabov, Ya. M. Kalychak, J. Alloys Comp., 330-332 (2002) 132.

[7] S. F. Matar, B. Chevalier, V. Eyert, J. Etourneau, Solid State Sci., 5 (2003) 1385.

[8] P. Raj, A. Sathyamoorthy, K. Shashikala, C. R. Venkateswara Rao, D. Kundaliya, S. K. Malik, J. Alloys Comp., 345 (2002) L1.

[9] S. K. Malik, D. Kundaliya, A. Sathyamoorthy, K. Shashikala, P. Raj, V. V. Krishnamurthy, J. Appl. Phys., 93 (2003) 7834.

[10] B. Chevalier, A. Wattiaux, J.-L. Bobet, J. Phys. : Condens. Matter, 18 (2006) 1743.

[11] P. Vajeeston, P. Ravindran, R. Vidya, A. Kjekshus, H. Fjellvag, V. A. Yartys, Phys. Rev. B, 67 (2003) 014101.

[12] Y. Bando, T. Suemitsu, K. Takagi, H. Tokushima, Y. Echizen, K. Katoh, K. Umeo, Y. Maeda, T. Takabatake, J. Alloys Comp., 313 (2000) 1.

[13] M. S. Kim, Y. Echizen, K. Umeo, S. Kobayashi, M. Sera, P. S. Salamakha, O. I. Sologub, T. Takabatake, X. Chen, T. Tayama, T. Sakakibara, M. H. Jung, M. B. Maple, Phys. Rev. B, 68 (2003) 054416.

[14] K. Latka, M. Rams, R. Kmiec, R. Kruk, A. W. Pacyna, T. Schmidt, G. Kotzyba, R. Pottgen, D. Johrendt, Acta Phys. Polonica B, 34 (2003) 1225.

[15] A. Slebarski, A. Jezierski, Phys. Stat. Sol. B, 236 (2003) 340.

[16] Ch. D. Routsi, J. K. Yakinthos, H. Gamari-Seale, J. Magn. Magn. Mater., 117 (1992) 79.

[17] A. Ślebarski, M. B. Maple, E. J. Freeman, C. Sirvent, M. Radłowska, A. Jezierski, E. Granado, Q. Huang, J. W. Lynn, Philos. Mag. B, 82 (2002) 943. 
[18] T. Schmidt, D. Johrendt, C. P. Sebastian, R. Pöttgen, K. Łątka, R. Kmieć, Z. Naturforsch., 60b (2005) 1036.

[19] P. Salamakha, O. Sologub, J. K. Yakinthos, Ch. D. Routsi, J. Alloys Comp., 265 (1998) L1.

[20] P. Rogl, B. Chevalier, M. J. Besnus, J. Etourneau, J. Magn. Magn. Mater., 80 (1989) 305.

[21] B. Chevalier, B. Malaman, Solid State Commun., 130 (2004) 711.

[22] E. Gaudin, B. Chevalier, B. Heying, U. Ch. Rodewald, R. Pöttgen, Chem. Mater., 17 (2005) 2693.

[23] D. Niepmann, Yu. M. Prots', R. Pöttgen, W. Jeitschko, J. Solid State Chem., 154 (2000) 329.

[24] Yu. M. Prots', R. Pöttgen, D. Niepmann, M. Wolff, W. Jeitschko, J. Solid State Chem., 142 (1999) 400.

[25] R. Kraft, R. Pöttgen, D. Kaczorowski, Chem. Mater., 15 (2003) 2998.

[26] R. Pöttgen, Th. Gulden, A. Simon, GIT Labor-Fachzeitschrift 43 (1999) 133.

[27] K. Yvon, W. Jeitschko, E. Parthé, J. Appl. Crystallogr., 10 (1977) 73.

[28] J.-L. Bobet, S. Pechev, B. Chevalier, B. Darriet, J. Alloys Comp., 267 (1998) 136.

[29] P. Dordor, E. Marquestaut, G. Villeneuve, Rev. Phys. Appl., 15 (1980) 1607.

[30] G. M. Sheldrick, SHELXL-97, Program for Crystal Structure Refinement, University of Göttingen, Germany, 1997.

[31] H. D. Flack, G. Bernadinelli, Acta Crystallogr., 55A (1999) 908.

[32] H. D. Flack, G. Bernadinelli, J. Appl. Crystallogr., 33 (2000) 1143.

[33] P. I. Krypyakevich, V. Ya. Markiv, E. V. Melnyk, Dopov. Akad. Nauk. Ukr. RSR, Ser. A (1967) 750.

[34] A. E. Dwight, M. H. Mueller, R. A. Conner, Jr., J. W. Downey, H. Knott, Trans. Met. Soc. AIME 242 (1968) 2075.

[35] M. F. Zumdick, R.-D. Hoffmann, R. Pöttgen, Z. Naturforsch., 54b (1999) 45.

[36] M. F. Zumdick, R. Pöttgen, Z. Kristallogr., 214 (1999) 90.

[37] A. E. Dwight, W. C. Harper, C. W. Kimball, J. Less-Common Met. 30 (1973) 1.

[38] R. Mishra, R. Pöttgen, R.-D. Hoffmann, H. Trill, B. D. Mosel, H. Piotrowski, M. F. Zumdick, Z. Naturforsch. 56b (2001) 589.

[39] J. Emsley, The Elements, Clarendon Press, Oxford (1989).

[40] J. Donohue, The Structures of the Elements, Wiley, New York (1974). 
[41] M. Sen, S. Giri, K. Ghoshray, B. Bandyopadhyay, A. Ghoshray, N. Chatterjee, Solid State Commun., 89 (1994) 327.

[42] J. A. Mydosh, Spin Glass : An Experimental Introduction (Taylor and Francis, London, 1993).

[43] B. Cornut, B. Coqblin, Phys. Rev. B, 5 (1972) 4541.

[44] D. Jaccard, J. Flouquet, J. Sierro, J. Appl. Phys., 57 (1985) 3084.

[45] V. Zlatic, B. Horvatic, I. Milat, B. Coqblin, G. Czycholl, C. Grenzebach, Phys. Rev. B, 68 (2003) 104432.

[46] V. Zlatic, I. Milat, B. Coqblin, G. Czycholl, Physica B, 312-313 (2002) 171.

[47] D. Huo, K. Mori, T. Kuwai, S. Fukuda, Y. Isikawa, J. Sakurai, Physica B, 281-282 (2000) 101.

[48] P. Link, D. Jaccard, P. Lejay, Physica B, 225 (1996) 207.

[49] D. Niepmann, R. Pöttgen, B. Künnen, G. Kotzyba, B. D. Mosel, Chem. Mater. 12 (2000) 533.

[50] R. Müllmann, U. Ernet, B. D. Mosel, H. Eckert, R. K. Kremer, R.-D. Hoffmann, R. Pöttgen, J. Mater. Chem. 11 (2001) 1133.

[51] Zh. Wu, B. D. Mosel, H. Eckert, R.-D. Hoffmann, R. Pöttgen, Chem. Eur. J. 10 (2004) 1558 .

[52] R.-D. Hoffmann, D. Kußmann, U. Ch. Rodewald, R. Pöttgen, C. Rosenhahn, B. D. Mosel, Z. Naturforsch. 54b (1999) 709. 
Table 1

Crystal data and structure refinement for CeIrSn, ZrNiAl type, space group $P \overline{6} 2 m, Z=3$.

\begin{tabular}{ll}
\hline Empirical formula & CeIrSn \\
Molar mass & $451.01 \mathrm{~g} / \mathrm{mol}$ \\
Unit cell dimensions & $a=743.8(2) \mathrm{pm}$ \\
& $c=407.58(7) \mathrm{pm}$ \\
& $\mathrm{V}=0.1953 \mathrm{~nm}^{3}$ \\
Calculated density & $11.51 \mathrm{~g} / \mathrm{cm}^{3}$ \\
Crystal size & $25 \times 25 \times 35 \mu^{3}$ \\
Transm. ratio (max/min) & $0.536 / 0.225$ \\
Absorption coefficient & $77.3 \mathrm{~mm}^{-1}$ \\
F(000) & 555 \\
$\theta$ range & $3^{\circ}$ to $35^{\circ}$ \\
Range in $h k l$ & $\pm 11, \pm 11, \pm 6$ \\
Total no. reflections & 3438 \\
Independent reflections & $352\left(\mathrm{R}_{\text {int }}=0.0816\right)$ \\
Reflections with $\mathrm{I}>2 \sigma(\mathrm{I})$ & $347\left(\mathrm{R}_{\text {sigma }}=0.0307\right)$ \\
Data /parameters & $352 / 14$ \\
Goodness-of-fit on $\mathrm{F}^{2}$ & 1.144 \\
Final R indices $[\mathrm{I}>2 \sigma(\mathrm{I})]$ & $\mathrm{R} 1=0.0171 ; w \mathrm{R} 2=0.0306$ \\
R indices (all data) & $\mathrm{R} 1=0.0177 ; w \mathrm{R} 2=0.0308$ \\
Extinction coefficient & $0.0142(6)$ \\
Flack parameter & $0.01(1)$ \\
Largest diff. peak and hole & $1.73 \mathrm{and}-1.18 \mathrm{e} / \AA^{3}$ \\
\hline
\end{tabular}

Table 2

Atomic coordinates and anisotropic displacement parameters $\left(\mathrm{pm}^{2}\right)$ for CeIrSn. $\mathrm{U}_{\mathrm{eq}}$ is defined as one third of the trace of the orthogonalized $U_{i j}$ tensor. The anisotropic displacement factor exponent takes the form: $-2 \pi^{2}\left[\left(h a^{*}\right)^{2} \mathrm{U}_{11}+\ldots+2 h k a^{*} b^{*} \mathrm{U}_{12}\right] . \mathrm{U}_{13}=\mathrm{U}_{23}=0$.

\begin{tabular}{lccccccccc}
\hline $\begin{array}{c}\text { Atom Wyckoff } \\
\text { site }\end{array}$ & $\mathrm{x}$ & $\mathrm{y}$ & $\mathrm{z}$ & $\mathrm{U}_{11}$ & $\mathrm{U}_{22}$ & $\mathrm{U}_{33}$ & $\mathrm{U}_{12}$ & $\mathrm{U}_{\mathrm{eq}}$ \\
\hline $\mathrm{Ce}$ & $3 f$ & $0.58651(9)$ & 0 & 0 & $45(2)$ & $58(3)$ & $70(2)$ & $29(1)$ & $56(1)$ \\
$\mathrm{Ir} 1$ & $2 d$ & $2 / 3$ & $1 / 3$ & $1 / 2$ & $68(1)$ & $\mathrm{U}_{11}$ & $109(2)$ & $34(1)$ & $82(1)$ \\
$\mathrm{Ir} 2$ & $1 a$ & 0 & 0 & 0 & $56(2)$ & $\mathrm{U}_{11}$ & $54(2)$ & $28(1)$ & $55(1)$ \\
$\mathrm{Sn}$ & $3 g$ & $0.25151(9)$ & 0 & $1 / 2$ & $47(2)$ & $53(3)$ & $65(3)$ & $27(2)$ & $55(1)$ \\
\hline
\end{tabular}


Table 3

Interatomic distances $(\mathrm{pm})$, calculated with the powder lattice parameters in the structure of CeIrSn. Standard deviations are equal or less than $0.2 \mathrm{pm}$. All distances of the first coordination spheres are listed.

\begin{tabular}{llllrlr}
\hline Ce: & 4 & Ir1 & 302.9 & Ir2: 6 & Sn & 276.6 \\
& 1 & Ir2 & 307.6 & 3 & Ce & 307.6 \\
& 2 & Sn & 321.9 & Sn: 2 & Ir2 & 276.6 \\
& 4 & Sn & 337.0 & 2 & Ir1 & 283.3 \\
& 4 & Ce & 388.2 & 2 & Ce & 321.9 \\
& 2 & Ce & 407.6 & 2 & Sn & 324.0 \\
Ir1: & 3 & Sn & 283.3 & 4 & $\mathrm{Ce}$ & 337.0 \\
& 6 & Ce & 302.9 & & &
\end{tabular}

Table 4. Crystallographic data relative to CeRhSn, CeIrSn and their hydrides.

\begin{tabular}{ccccccc}
\hline Compound & $a(\mathrm{pm})$ & $\Delta a / a(\%)$ & $c(\mathrm{pm})$ & $\Delta c / c(\%)$ & $\mathrm{V}\left(\mathrm{nm}^{3}\right)$ & $\Delta \mathrm{V} / \mathrm{V}(\%)$ \\
\hline $\mathrm{CeRhSn}$ & $744.8(2)$ & & $408.00(9)$ & & 0.1960 & \\
$\mathrm{CeRhSnH}_{0.8}$ & $755.7(2)$ & 1.5 & $413.6(1)$ & 1.4 & 0.2046 & 4.4 \\
$\mathrm{CeIrSn}$ & $743.8(2)$ & & $407.58(7)$ & & 0.1953 & \\
$\mathrm{CeIrSnH}_{0.7}$ & $755.5(2)$ & 1.6 & $413.8(1)$ & 1.5 & 0.2045 & 4.7 \\
\hline
\end{tabular}


Table 5

Fitting parameters of ${ }^{119} \mathrm{Sn}$ Mössbauer measurements in CeRhSn, $\mathrm{CeRhSnH}_{0.8}$, $\mathrm{CeIrSn}$, and $\mathrm{CeIrSnH}_{0.7}$. Numbers in parentheses represent the statistical errors in the last digit. $\delta=$ isomer shift, $\Delta \mathrm{E}_{\mathrm{Q}}=$ electric quadrupole interaction, $\Gamma=$ experimental line width.

\begin{tabular}{|c|c|c|c|c|}
\hline Compound & $\delta(\mathrm{mm} / \mathrm{s})$ & $\Delta \mathrm{E}_{\mathrm{Q}}(\mathrm{mm} / \mathrm{s})$ & $\Gamma(\mathrm{mm} / \mathrm{s})$ & $\chi^{2}$ \\
\hline \multicolumn{5}{|l|}{$78 \mathrm{~K}$ data } \\
\hline CeRhSn & $1.84(7)$ & $1.12(8)$ & $0.94(6)$ & $0.96(5)$ \\
\hline $\mathrm{CeRhSnH}_{0.8}$ & $1.87(2)$ & $1.28(3)$ & $0.97(6)$ & $1.06(9)$ \\
\hline CeIrSn & $1.76(3)$ & - & $1.16(8)$ & $1.34(5)$ \\
\hline $\mathrm{CeIrSnH}_{0.7}$ & $1.85(1)$ & $1.06(3)$ & $1.02(8)$ & $1.19(3)$ \\
\hline \multicolumn{5}{|l|}{$4.2 \mathrm{~K}$ data } \\
\hline CeRhSn & $1.83(4)$ & $1.10(2)$ & $0.94(2)$ & $0.93(1)$ \\
\hline $\mathrm{CeRhSnH}_{0.8}$ & $1.86(3)$ & $1.26(7)$ & $1.03(1)$ & $1.08(1)$ \\
\hline CeIrSn & $1.75(4)$ & - & $1.19(1)$ & $1.15(2)$ \\
\hline $\mathrm{CeIrSnH}_{0.7}$ & $1.85(6)$ & $1.08(1)$ & $1.07(2)$ & $1.16(2)$ \\
\hline
\end{tabular}




\section{Figure captions}

Fig. 1. Unit cell volume of the hydrides $\mathrm{CeMXH}_{\mathrm{y}}$ crystallizing in the $\mathrm{ZrNiAl}$-type versus the unit cell volume of intermetallics CeMX. The dashed line presents the linear fit of the data.

Fig. 2. Temperature dependence of the reciprocal magnetic susceptibility of CeRhSn (a), CeIrSn (b) and their hydrides measured at the applied magnetic field $\mu_{0} \mathrm{H}=4 \mathrm{~T}$. The dashed lines represent fits to the data of $\chi_{\mathrm{m}}=\chi_{0}+\mathrm{C} /\left(\mathrm{T}-\theta_{\mathrm{p}}\right)$ for CeRhSn and CeIrSn and $\chi_{\mathrm{m}}=\mathrm{C} /\left(\mathrm{T}-\theta_{\mathrm{p}}\right)$ for $\mathrm{CeRhSnH}_{0.8}$ and $\mathrm{CeIrSnH}_{0.7}$.

Fig. 3. Temperature dependence at $\mu_{0} \mathrm{H}=0.1 \mathrm{~T}$ of the zero-field-cooled (ZFC) and fieldcooled (FC) magnetic susceptibility of hydride $\mathrm{CeRhSnH}_{0.8}$.

Fig. 4. Temperature dependence of the reduced electrical resistivity of $\mathrm{CeRhSnH}_{0.8}$ and $\mathrm{CeIrSnH}_{0.7}$. The inset shows the plot for $\mathrm{CeRhSnH}_{0.8}$ at $\mathrm{T}<50 \mathrm{~K}$.

Fig. 5. Temperature dependence of the thermoelectric power for hydrides $\mathrm{CeRhSnH}_{0.8}$ and $\mathrm{CeIrSnH}_{0.7}$.

Fig. 6. Experimental and simulated ${ }^{119} \mathrm{Sn}$ Mössbauer spectra of CeRhSn and $\mathrm{CeRhSnH}_{0.8}$ at 78 K. For details see Table 5 and text.

Fig. 7. Experimental and simulated ${ }^{119} \mathrm{Sn}$ Mössbauer spectra of CeIrSn and $\mathrm{CeIrSnH}_{0.7}$ at 78 K. For details see Table 5 and text. 


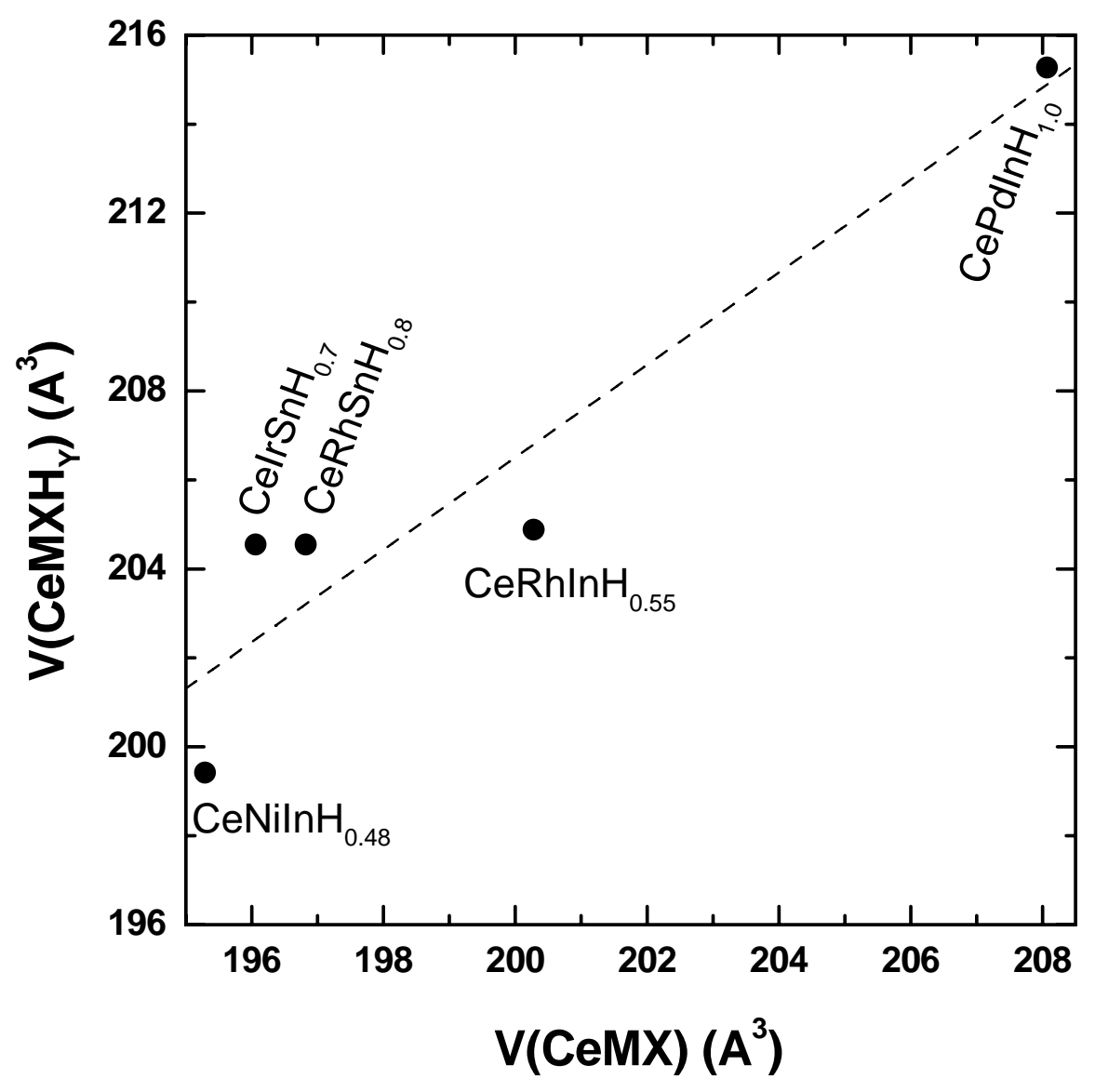

Fig. 1. Unit cell volume of the hydrides $\mathrm{CeMXH}_{\mathrm{y}}$ crystallizing in the $\mathrm{ZrNiAl}$-type versus the unit cell volume of intermetallics CeMX. The dashed line presents the linear fit of the data. 

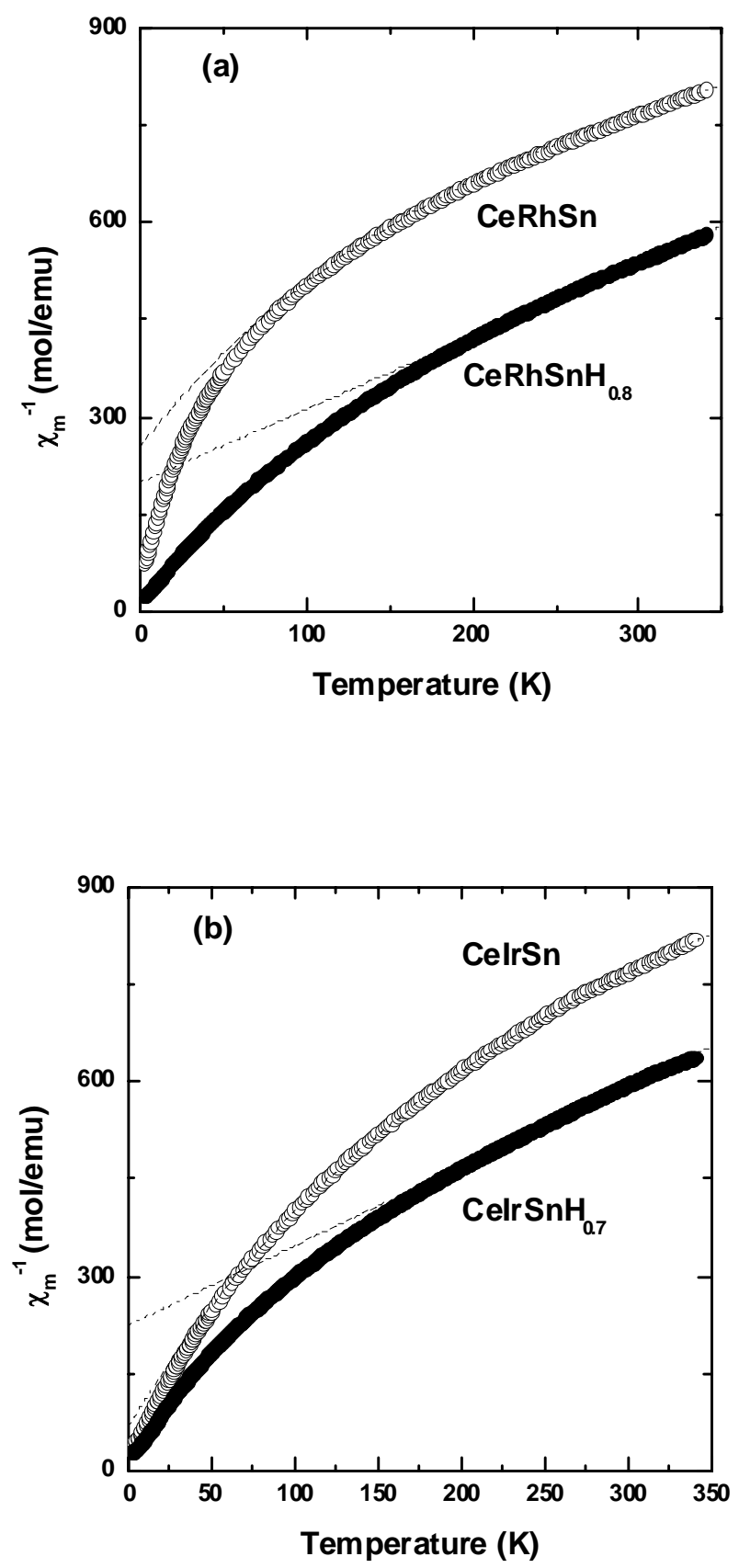

Fig. 2. Temperature dependence of the reciprocal magnetic susceptibility of CeRhSn (a), CeIrSn (b) and their hydrides measured at the applied magnetic field $\mu_{0} \mathrm{H}=4 \mathrm{~T}$. The dashed lines represent fits to the data of $\chi_{\mathrm{m}}=\chi_{0}+\mathrm{C} /\left(\mathrm{T}-\theta_{\mathrm{p}}\right)$ for CeRhSn and CeIrSn and $\chi_{\mathrm{m}}=\mathrm{C} /\left(\mathrm{T}-\theta_{\mathrm{p}}\right)$ for $\mathrm{CeRhSnH}_{0.8}$ and $\mathrm{CeIrSnH}_{0.7}$. 


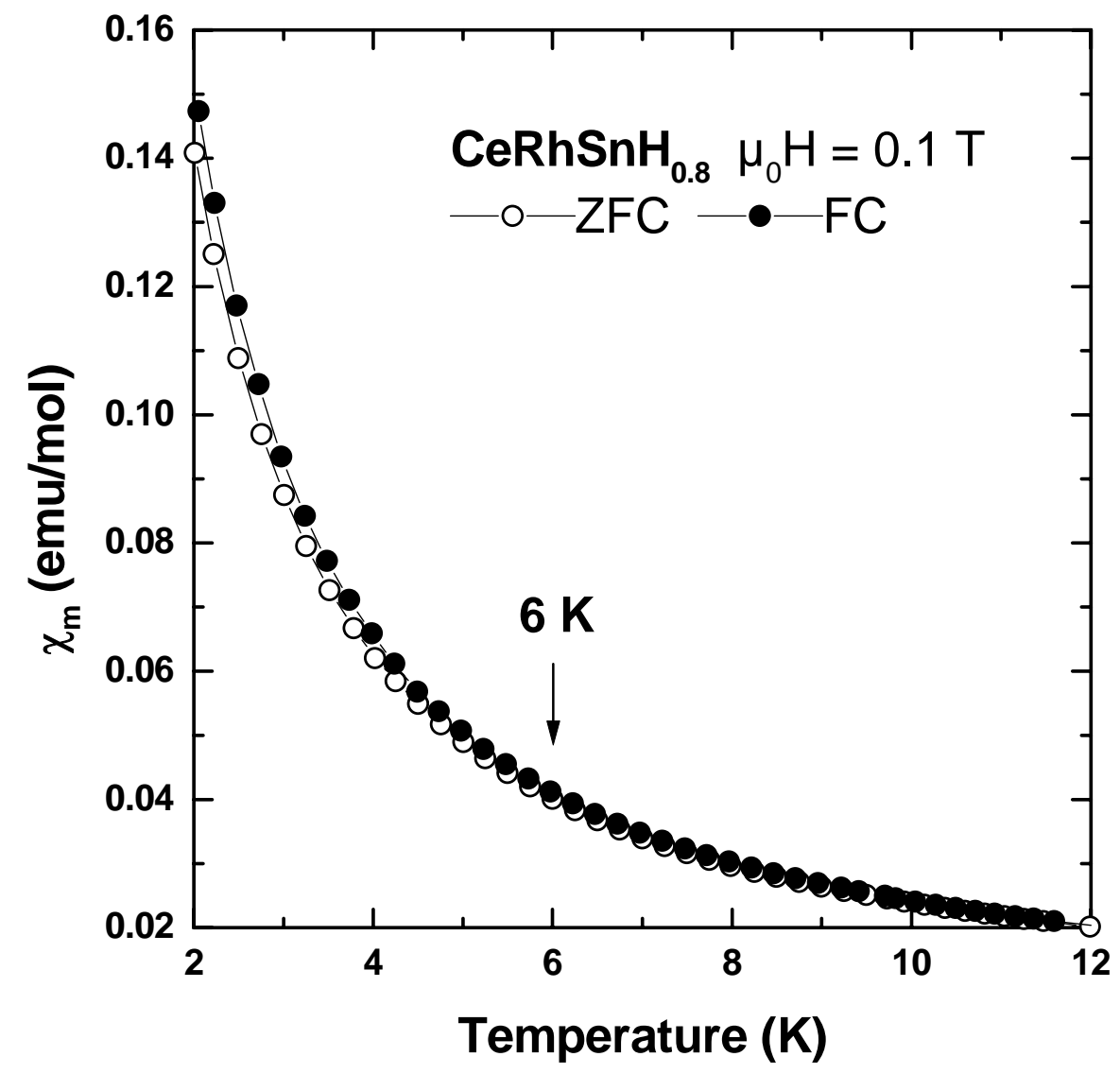

Fig. 3. Temperature dependence at $\mu_{0} \mathrm{H}=0.1 \mathrm{~T}$ of the zero-field-cooled (ZFC) and fieldcooled (FC) magnetic susceptibility of hydride $\mathrm{CeRhSnH}_{0.8}$. 


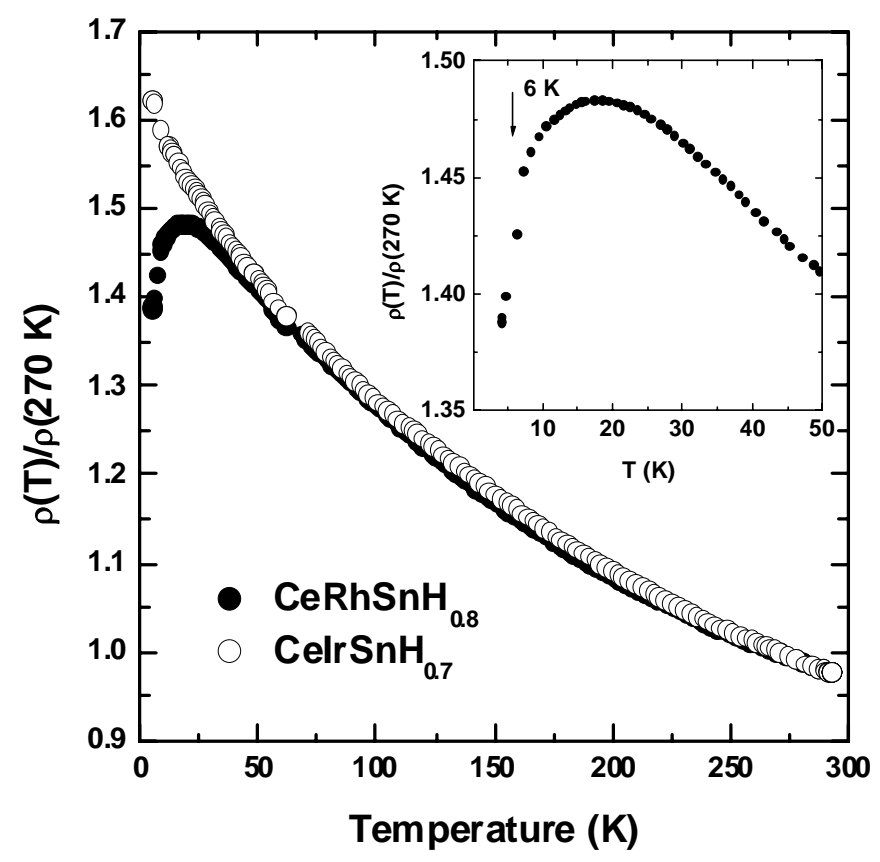

Fig. 4. Temperature dependence of the reduced electrical resistivity of $\mathrm{CeRhSnH}_{0.8}$ and $\mathrm{CeIrSnH}_{0.7}$. The inset shows the plot for $\mathrm{CeRhSnH}_{0.8}$ at $\mathrm{T}<50 \mathrm{~K}$. 


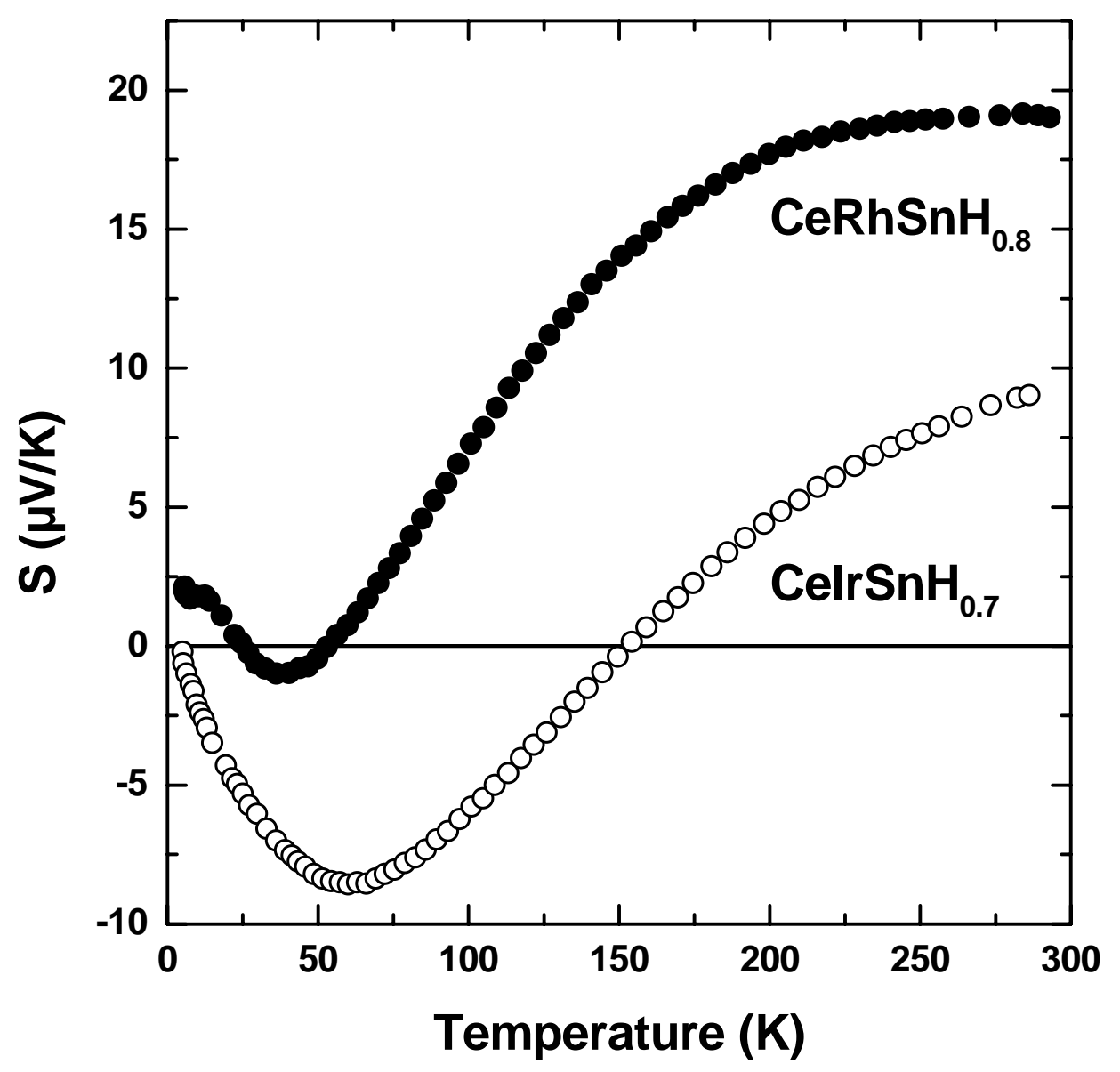

Fig. 5. Temperature dependence of the thermoelectric power for hydrides $\mathrm{CeRhSnH}_{0.8}$ and $\mathrm{CeIrSnH}_{0.7}$. 


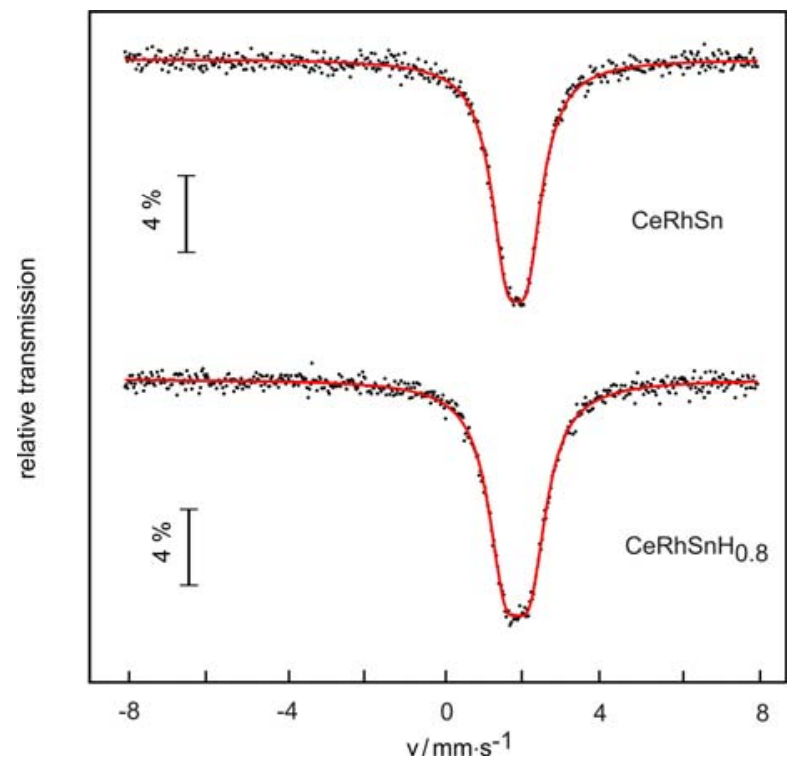

Fig. 6. Experimental and simulated ${ }^{119} \mathrm{Sn}$ Mössbauer spectra of $\mathrm{CeRhSn}$ and $\mathrm{CeRhSnH}_{0.8}$ at 78 K. For details see Table 5 and text.

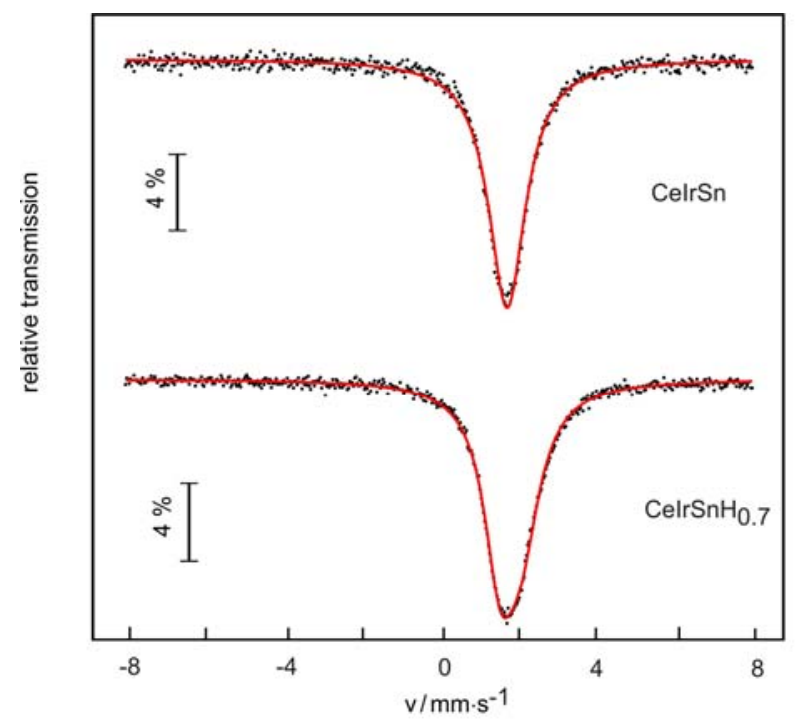

Fig. 7. Experimental and simulated ${ }^{119} \mathrm{Sn}$ Mössbauer spectra of CeIrSn and $\mathrm{CeIrSnH}_{0.7}$ at 78 K. For details see Table 5 and text. 